\title{
Inklusion und kinderrechtsorientierte Schulentwicklung
}

Lothar Krappmann

\section{Zusammenfassung}

Die Behindertenrechtskonvention der Vereinten Nationen hat der Aufnahme von Kindern mit Behinderungen in Regelschulen großen Nachdruck gegeben. Es reicht jedoch nicht, Barrieren abzubauen und das Lernen dieser Kinder individuell zu unterstützen. Sie müssen eine Schule erleben können, in der alle Kinderrechte verwirklicht werden, die die Staaten den Kindern in der Kinderrechtskonvention zugesichert haben. Darin liegt eine herausfordernde Aufgabe für die Weiterentwicklung unserer Schulen. Denn nur in einer Schule, die Bildung und alltägliches Schulleben auf den Menschenrechten gründet, kann Inklusion aller in gemeinsames Leben verwirklicht werden. Entscheidend ist, dass alle Kinder, auch Kinder mit Behinderung, mit ihren Sichtweisen, Meinungen und Interessen anerkannt und befähigt werden, an Entscheidungen über gemeinsame Angelegenheiten mitzuwirken.

Die UN-Behindertenrechtskonvention (im Folgenden UN-BRK), seit 2008 in Deutschland in Kraft, hat mit völkerrechtlicher Autorität und allem Nachdruck ihrer Bestimmungen klargestellt, dass die Bildungsstätten der Vertragsstaaten auch die Kinder aufzunehmen haben, die wegen einer Behinderung einer besonderen Unterstützung bedürfen, um sich erfolgreich entwickeln und bilden zu können (Kinder sind für UN-Dokumente alle jungen Menschen bis 18). ${ }^{1}$ Dieses Menschen-

1 Die Texte der Behindertenrechtskonvention, der Kinderrechtskonvention und der weiteren Menschenrechtsverträge mit samt begleitender Materialien sind auf den Internet-Seiten des Deutschen Instituts für Menschenrechte zu finden: http://www.institut-fuer-men- 
recht der Kinder (und Jugendlichen) mit Behinderungen wird zwar immer noch in der Öffentlichkeit diskutiert, dennoch hat die UN-BRK massive Anstrengungen ausgelöst, Kinder mit Behinderungen in öffentlichen Regelschulen zu unterrichten, wie es Eltern, Pädagog*innen und auch Kinder mit Behinderungen selber seit Jahrzehnten verlangen.

\section{Inklusion der Kinder mit Behinderungen nach den UN-Menschenrechtsverträgen}

Wie schwer es politischen Kräften und amtlichen Stellen fällt, sich auf die volle Teilhabe der Menschen mit Behinderungen an allen gesellschaftlichen Prozessen einzustellen, ist daraus abzulesen, dass die deutsche Übersetzung der UN-BRK den in der Menschenrechts-Community seit der UNESCO-Konferenz in Salamanca 1994 durchgängig verwendeten Begriff der inclusion vermeidet. ${ }^{2}$ In der UN-Kinderrechtskonvention, die in den 80er-Jahren ausgearbeitet wurde, kam dieser Begriff noch nicht vor (im Folgenden: UN-KRK). ${ }^{3}$ Dieser Begriff, der ausdrückt, dass die Menschenrechte mehr als bloßes Dabei-Sein fordern, war noch nicht gebräuchlich. Es besteht jedoch kein Zweifel, dass die Rechte der Kinderrechtskonvention ausnahmslos auch für Kinder mit Behinderungen gelten. Die UN-KRK war sogar der erste Menschenrechtsvertrag, der jegliche Diskriminierung von Kindern aufgrund einer Behinderung ausdrücklich untersagte. Sie enthält einen eigenen Artikel über die Rechte der Kinder mit Behinderungen auf „aktive Teilhabe " und „vollständige soziale Integration" (Art. 23, Abs. 1 und 3).

Der Begriff der Inklusion fand bald in Stellungnahmen und Forderungen Verbreitung, um der Forderung nach effektiver Beteiligung von Personen mit Behinderungen am gesellschaftlichen Leben Ausdruck zu verleihen. Folglich wurde er auch vom UN-Kinderrechtsausschuss in seinen Kommentar Nr. 9 ,Die Rechte von Kindern

schenrechte.de. Viele Ministerien und Nichtregierungsorganisationen bieten ebenfalls die Texte und Kommentierungen.

2 The Salamanca statement and framework for action on special needs education: http:// unesdoc.unesco.org/images/0009/000984/098427eo.pdf. Zugegriffen: 29.01.2017.

3 Die Kinderrechtskonvention umfasst die wirtschaftlichen, sozialen und kulturellen sowie die politischen und bürgerlichen Rechte der Kinder. Weltweit ist die Kinderrechtskonvention auch deswegen ein wichtiger Bezugspunkt für Kinder mit Behinderungen und ihre Unterstützer, weil die Kinderrechtskonvention von 196 Staaten ratifiziert wurde, die Behindertenrechtskonvention bislang erst von 166 Staaten (August 2016). Siehe http:// indicators.ohchr.org/ 
mit Behinderungen' übernommen (veröffentlicht 2006 kurz vor der Verabschiedung der UN-BRK). In diesem Kommentar wies der UN-Kinderrechtsausschuss darauf hin, dass zur Verwirklichung des Ziels der Inklusion wirksame Maßnahmen im Bildungswesen getroffen werden müssen (Abs. 12 des Kommentars). Was dafür geschehen muss, führte die UN-BRK in ihrem umfassenden Artikel 24 weiter aus.

In diesem Aufsatz will ich die menschenrechtliche Aufgabe, Inklusion im Bildungswesen zu fördern und herzustellen, aus einer Sicht betrachten, die in den Bildungsartikeln der beiden Konventionen angelegt ist, aber in der Praxis der Umsetzung der Artikel nicht mit Konsequenz verfolgt wird. Diese Umsetzungspraxis geht offensichtlich davon aus, dass die Bildungsstätten, in die Kinder mit Behinderungen inkludiert und zur aktiven Teilhabe am sozialen, beruflichen und bürgerlichen Leben befähigt werden sollen, abgesehen von einigen Vorkehrungen und Unterstützungsleistungen für Kinder mit Behinderungen, so weiterarbeiten können wie bisher. Sicherlich sollen inklusive Schulen gute Schulen sein. Aber es ist nicht zu vernehmen, dass diese Schulen aus Menschenrechtssicht überdacht und ihr Bildungskanon und ihre Unterrichtsweisen geprüft und weiterentwickelt werden müssten.

In jüngeren Äußerungen von UN-Gremien über das Recht auf Bildung wird zwar des Öfteren betont, dass quality education angeboten werden müsse, so etwa in dem 2015 von der UN-Generalversammlung verabschiedeten Ziel 4 für nachhaltige Entwicklung „Ensure inclusive and quality education for all“ ${ }^{4}$ Was quality education einschließt, wird jedoch nicht weiter ausgeführt. Vermutlich denkt man an didaktisch gut angelegten Unterricht, der von gut ausgebildeten Lehrkräften erteilt wird.

Einen anderen Ton setzt der Ausschuss für die Wirtschaftlichen, Sozialen und Kulturellen Rechte, der die Einhaltung des UN-Sozialpakts überwacht. ${ }^{5}$ Der Sozialpakt, in Kraft seit 1976, enthält zwei Artikel über das Menschenrecht auf Bildung (Art. 13 und 14). Im Kommentar des Ausschusses zu Artikel 13 verlangt der Ausschuss, dass die den Kindern zu bietende Bildung sich sowohl den Bedürfnissen der sich wandelnden Gesellschaft als auch der Lebenssituation der Lernenden innerhalb der verschiedenartigen sozialen und kulturellen Umfelder anpassen kann, und zwar auf allen Stufen des Bildungswesens (adaptability; siehe insbesondere

4 Siehe die Internetseite: http://www.un.org/sustainabledevelopment/education/. Zugegriffen: 29.01.2017.

5 Internationaler Pakt über wirtschaftliche, soziale und kulturelle Rechte (kurz: UN-Sozialpakt). http://www.sozialpakt.info/internationaler-pakt-ueber-wirtschaftliche-soziale-und-kulturelle-rechte-3111/. Zugegriffen: 29.01.2017. 
die Absätze 6 und 50 des Kommentars). ${ }^{6}$ Der Kommentar konkretisiert nicht, wie Schulen sich dieser Aufgabe widmen sollen. Aber sicher ist, dass Schulen sich mit ihren Curricula und Arbeitsweisen stets weiterentwickeln müssen, um mit ihren Angeboten auf gesellschaftliche Erfordernisse und Lebensinteressen der jungen Menschen zu antworten. Inklusion gehört zu den exemplarischen Herausforderungen von adaptability.

Tatsächlich haben die Staaten mit ihrem Beitritt zur UN-KRK und zur UN-BRK zugesagt, eine weit über Fachunterricht hinausreichende Bildungsvorstellung in ihren Schulen zu verwirklichen. ${ }^{7}$ Die Bildungseinrichtungen sollen dazu beitragen, dass alle Menschen ihr Leben auf der Grundlage der unverlierbaren Rechte aller Menschen führen können: in gegenseitiger Achtung, ohne Diskriminierung, im gemeinsamen Bemühen um friedliche Konfliktlösung und gerechte Lebensverhältnisse und Beteiligung aller an den sie betreffenden Angelegenheiten.

Diese Grundausrichtung müsste die Arbeit inklusiver Bildungsstätten rahmen, denn Inklusion betrifft Menschen mit allen Rechten. Kaum jemand stellt jedoch diesen menschenrechtlichen Rahmen her. Stattdessen stehen kind- und unterrichtsnahe Probleme im Vordergrund: Sind Regelschulen erreichbar? Wurden Barrieren abgebaut? Steht genug Unterstützung für das Lernen bereit? Das sind zweifellos dringliche Fragen, bei denen jedoch aus dem Blick gerät, dass sowohl die UN-BRK als auch die UN-KRK in ihren Bildungsartikeln ausdrücklich auf mehr zielen, als erfolgreiche Teilnahme an gutem Fachunterricht sicherzustellen.

Drei Ziele beherrschen den UN-Diskurs über das Menschenrecht auf Bildung seit der Allgemeinen Erklärung der Menschenrechte von 1948: Förderung aller menschlichen Potentiale, umfassendes Bewusstsein der Rechte aller Menschen sowie menschenrechtliche Handlungsfähigkeit. Dieser dreigegliederten Aufschlüsselung des Menschenrechts auf Bildung folgen alle Menschenrechtsverträge. Sie ist Grundlage der Bildungsartikel der Kinderrechts- und der Behindertenrechtskonvention und muss auch der Bezugspunkt für inklusive Bildung sein. ${ }^{8}$

6 Committee on Economic, Social and Cultural rights (1999). General Comment No. 13: The right to education.

7 Die UN-Generalversammlung berät und beschließt die Menschenrechtsverträge, Pakte oder Konventionen genannt, deren Bestimmungen aber erst durch die aus eigenem Entschluss erfolgende Ratifikation in die Rechtssysteme der einzelnen Staaten übernommen wird. Sie verpflichten sich, die Bestimmungen der Konvention rechtlich und in der Praxis umzusetzen.

8 Da die Allgemeine Erklärung der Menschenrechte „nur“ eine Erklärung der UN-Vollversammlung war, die rechtlich nicht verbindlich ist, werden die allen Menschen zuerkannten Rechte dieser Erklärung durch Menschenrechtsverträge zu einklagbaren Rechten gemacht (vgl. Cremer 2011). 


\section{Die drei Ziele einer menschenrechtlich begründeten Bildung}

\subsection{Erstes Bildungsziel: Ganzheitliche Bildung}

Für alle Kinder: Zum Ersten vereinbarten die Staaten, die Bildung des Kindes darauf auszurichten, „die Persönlichkeit, die Begabung und die geistigen und körperlichen Fähigkeiten des Kindes voll zur Entfaltung zu bringen“"(Artikel 29 Abs. 1 (a) der UN-KRK). In Artikel 24, Abs. 1 (b) der UN-BRK wird diese Zielsetzung fast wörtlich wiederholt: Die Vertragsstaaten gewährleisten Menschen mit Behinderungen „ein [inklusives] Bildungssystem auf allen Ebenen (...) mit dem Ziel, zu gewährleisten, Menschen mit Behinderungen ihre Persönlichkeit, ihre Begabungen und ihre Kreativität sowie ihre geistigen und körperlichen Fähigkeiten voll zur Entfaltung bringen zu lassen."

Die Bildungswissenschaften bezeichnen eine Bildung mit diesem umfassenden Spektrum von Bildungsbereichen üblicherweise als ganzheitliche Bildung. In der Realität der meisten Schulen gibt es bestenfalls Ansätze zu einer derartigen ganzheitlichen Bildung; einige nicht primär kognitiv ausgerichtete Fächer ergänzen die Kernfächer. Der Begriff Nebenfächer signalisiert deren Stellung. Manche Angebote sind optional, oft auf den Nachmittag verlagert. Das Grundanliegen einer Bildung, die alle Fähigkeiten des Menschen entfaltet, wird verfehlt. Eltern und Öffentlichkeit sehen die Schule als eine Einrichtung, die intellektuelle Schulleistungen hervorbringen soll; alles andere gilt als schönes Beiwerk.

Mit Blick auf Kinder mit Behinderungen: Eine engspurige Bildung beeinträchtigt alle Kinder. Ganz besonders schadet sie jedoch der Entwicklung der Kinder und späteren Erwachsenen mit Behinderungen. Sie sind vielen Situationen, Erlebnissen und Widerfahrnissen ausgesetzt, die nicht nur kognitiv bearbeitet werden können. Sie brauchen Zugang zu den unterschiedlichen Dimensionen der Sinn- und Selbstfindung, zu den vielfältigen Wegen der Verständigung mit anderen und zu den Problembearbeitungen, die Menschen in Spiel, Theater, Kunst, Musik, Tanz, Design oder Meditation zur Verfügung stehen. Diese Erweiterung menschlicher Entwicklungsmöglichkeiten über spezifische Fachbildung hinaus kann nicht zufälligen Gegebenheiten überlassen werden, sondern braucht einen festen Ort auf dem Bildungsweg aller Kinder - erst recht in der inklusiven Schule.

9 Wie erwähnt, steht in der amtlichen nicht-verbindlichen deutschen Übersetzung das Wort integrativ für den Begriff inclusive im völkerrechtlich verbindlichen englischen Vertragstext. 
Mit umfassend entwickelten Fähigkeiten am Leben teilnehmen zu können ist auch für die Gestaltung menschlicher Gemeinschaft erforderlich, denn Kooperation mit anderen verlangt, nicht nur kognitiv aufmerksam zu sein, sondern alle Möglichkeiten des Gesprächs, der Auseinandersetzung, der Problemlösung und der emotionalen Beteiligung ausschöpfen zu können, um sinnvolle, gerechte, menschenfreundliche und nachhaltige Lebensformen zu entwickeln. Wiederum ist für Kinder mit Behinderungen wichtig, sich in einem Schulumfeld entwickeln und bilden zu können, das diese umfassende Erfahrung mit Menschen, Sachen, Sinn und Werten anbietet.

\subsection{Zweites Bildungsziel: Bewusstsein der Rechte aller Menschen}

Für alle Kinder: Zum Zweiten stellen die Vertragsstaaten beider Konventionen ihren Schulen die Aufgabe, Kindern bewusst zu machen, dass die Menschenrechte verbindliche Grundlage des Zusammenlebens aller Menschen sein müssen. In der UN-BRK steht: Das inklusive Bildungssystem habe „das Bewusstsein der Würde und das Selbstwertgefühl des Menschen“ voll zu entfalten und die „Achtung vor den Menschenrechten, den Grundfreiheiten und der menschlichen Vielfalt" zu stärken (Art. 24, Abs. 1(a)). Entsprechend formuliert die UN-KRK: Bildung müsse dem Kind „Achtung vor den Menschenrechten und Grundfreiheiten und den in der Charta der Vereinten Nationen verankerten Grundsätzen vermitteln“(Art. 29, Abs. 1 (b)).

Es sei daran erinnert, dass die Vereinten Nationen gegründet wurden, um eine friedliche, gerechte und freiheitliche Welt für alle Menschen ohne Diskriminierung zu schaffen. Die Allgemeine Erklärung der Menschenrechte von 1948 hat nach heftigen Auseinandersetzungen festgeschrieben, welche Rechte folglich Menschen niemals genommen werden dürfen. Es ist einseitig, die Vereinten Nationen vor allem mit Blick auf ihren Sicherheitsrat und dessen militärische und humanitäre Aktionen wahrzunehmen, mit denen sich dieses Gremium bemüht, militärische Konflikte und humanitäre Katastrophen zu entschärfen. Denn die erste Aufgabe der Weltorganisation ist eine präventive, nämlich den Aufbau einer Welt zu fördern, in der alle Menschen in wechselseitiger Achtung zusammenleben, ihre Konflikte friedlich lösen und Probleme in gerechtem Interessenausgleich bewältigen.

Ohne Bildung aller Menschen ist dies nicht vorstellbar, weil alle Menschen das Recht haben, ihre Auffassung über Gerechtigkeit und Frieden schaffende Lösungen einzubringen. Sie brauchen dafür Kenntnisse, Handlungsfähigkeit und Urteilsvermögen. Daher ist Bildung für die Anstrengungen der Vereinten Nationen unverzichtbar. Dies wird durch die fast gleichlautende Wiederholung der menschen- 
rechtlichen Zielsetzungen des Bildungswesens in den Menschenrechtsverträgen nachdrücklich unterstrichen.

Mit Blick auf Kinder mit Behinderungen: Wiederum ist zu betonen, dass Kinder mit Behinderungen besonders darauf angewiesen sind, in Schulen und Tagesstätten eine Bildung in sich aufzunehmen, die dem Zusammenleben aller Menschen eine Grundlage gibt. Seit unerdenklichen Zeiten wurden die Würde, die Rechte und eigene Entscheidungen dieser Menschen missachtet, wurden sie als einzelne und als Gruppe von Menschen an den Rand des sozialen, gesellschaftlichen und politischen Geschehens gedrängt. Sie müssen verstehen und darauf beharren können, was allen Menschen und auch ihnen in gleichem Maße verbürgt ist, nämlich Mensch mit Rechten und Freiheiten unter Menschen mit Rechten und Freiheiten zu sein. Sonst sind Wissen und Können aus dem Fachunterricht nur von eingeschränktem Nutzen.

Allerdings ist aus einer Studie von Bauer und Feibig (2013) zu folgern, dass eine reine Unterrichtung über zustehende Menschenrechte das Selbstbewusstsein von Kindern mit Behinderungen auch belasten kann, denn sie nehmen wahr, wie weit die soziale Realität hinter ihren Rechten zurückbleibt. Diese Kinder müssen Fortschritte in der Umsetzung ihrer Rechte erfahren, um das Bewusstsein zu entwickeln, dass die Menschenrechte verbindlich sind und den Einsatz lohnen. Schulleben muss bewusstseinsbildende Beweiskraft haben.

\subsection{Drittes Bildungsziel: Menschenrechtliche Handlungsfähigkeit}

Für alle Kinder: Konsequenterweise klären die Vertragsstaaten der UN-KRK und der UN-BRK eindeutig, dass bloße Information über Menschenrechte und ihre Umsetzungsprobleme nicht genügt. In Artikel 24, Absatz 1 (c), der UN-BRK versprechen die Staaten eine inklusive Bildung, die die Kinder mit Behinderungen „zur wirklichen Teilhabe an einer freien Gesellschaft befähig( $\mathrm{t})^{\text {" [ }}$ to participate effectively im englischen Originaltext]. Entsprechend heißt es in der Kinderrechtskonvention, die Bildung müsse darauf ausgerichtet sein, „das Kind auf ein verantwortungsbewusstes Leben in einer freien Gesellschaft vorzubereiten" (Art. 29, Abs. 1 (d)).

Es sind viele Probleme, die auf verantwortungsbewusste Bewältigung warten. Oft sind es die Kehrseiten von wissenschaftlichen und technischen Errungenschaften, die das Leben der Menschen erleichtern und neue Handlungsmöglichkeiten eröffnet haben. Desto dringender ist es, Lösungen zu finden, die die Vor- und Nachteile ausgleichen, ohne Ungerechtigkeiten und Exklusion zu erzeugen. 
So wichtig es ist, dass die Wissenschaften Erkenntnis und Verstehen mehren, so evident ist auch, dass Wissenschaft und Technik allein menschengerechtes Leben nicht sichern können. Die Bürger*innen des Gemeinwesens müssen aushandeln, welche Problemlösungen fair, gerecht, sozial verträglich sind, wie etwa langwierige Auseinandersetzungen über Abgase, Stromtrassen oder Plastikmüll demonstrieren. Erst recht gilt dies für Probleme wie soziale Ungleichheit, kulturelle Vielfalt oder Diskriminierung. Die Befähigung zur Bewältigung dieser Probleme ist eine genuine Bildungsaufgabe, die in die Schule gehört, um verantwortungsbewussten Umgang mit den Rechten aller Menschen zu sichern (Krappmann und Petry 2016).

Unsere Kultusminister*innen erkennen der Schule diese Aufgabe jedoch nicht zu. In ihrer Erklärung zur Menschenrechtsbildung (2000) ordnen sie den aktiven Einsatz für Menschenrechte, dem „persönlichen und politischen Lebensumkreis“ der Schüler*innen zu. In diesem Lebensumkreis außerhalb der Schule steht keine begleitende Unterstützung zur Verfügung, die Wissen klärt, auf Erfahrung verweist, kritisch nachfragt, Enttäuschung auffängt und vor Simplifizierung warnt. Beteiligung aller ist nicht garantiert.

Dass Erkennen, Verstehen und Handeln nicht zu trennen sind, demonstriert Nussbaum (2015) am Beispiel von Bildungsprozessen indischer Frauen in desolaten Landregionen. Diese Frauen entwickeln ihre kognitiven, sozialen und moralischen Fähigkeiten im Kampf um die Verbesserung ihrer Lebenssituation. Gewiss greift Handeln auf Vorkenntnis zurück. Aber da handelnde Menschen auf Bedingungen und Einflusskräfte stoßen, die neues Begreifen und Beurteilen hervorbringen, erweitert es wiederum die Fähigkeiten. Daher ist die Verbindung von Analyse und Verstehen mit Handeln für eine inklusive Schule wesensgemäße Aufgabe.

In allen Schulen gibt es Probleme, die Menschenrechte betreffen: Gewalt, Vorurteile, Herabsetzung. Alle Schulen stehen vor der Aufgabe, kulturelle Diversität konstruktiv zu nutzen, geflohene Kinder zu integrieren und, nicht zuletzt, Kinder mit Behinderungen zu inkludieren. Diese Aufgaben sind nicht administrativ zu regeln, sondern rufen nach gemeinsamer Analyse, Erfahrungsaustausch, Beurteilung und Verantwortungsübernahme durch Lehrkräfte, Kinder und Jugendliche. Das ist Vorbereitung auf verantwortungsbewusstes Leben durch volle Teilhabe im Lebensumkreis der Schule.

Mit Blick auf Kinder mit Behinderungen: Es wäre nicht zu verstehen, wenn eine Schule, die sich der Verpflichtung stellt, zur Umsetzung der Menschenrechte zu befähigen, die Inklusion der Kinder mit Behinderungen nicht als vorrangiges Thema aufgreifen würde. Zum einen ist dafür zu sorgen, dass die Kinder mit Behinderungen voll in Unterricht und Schulleben einbezogen werden. Dafür reicht nicht, Barrierefreiheit herzustellen und Lernhilfen zu organisieren. Schulleben ist 
weit mehr als Unterricht und Lernen. Mitwirkung in schulischen Gestaltungs- und Entscheidungsprozessen muss erreicht werden. Auch in Schulen mit einem kooperativen Sozialklima gehören Streit, Foppereien und Rangeleien unter Kindern und manch fragwürdige Regelverstöße dazu. Die besonderen Lebensbedingungen der Kinder mit Behinderungen müssen dabei stets beachtet werden (wie auch die der anderen Kinder), aber sie haben keinen menschenrechtlichen Sonderstatus. Volle Inklusion in das vielgestaltige Sozialleben der Schule zu erreichen, fordert allen Beteiligten Aufmerksamkeit, Perspektivenwechsel, Grenzsetzungen und Achtung menschlicher Würde ab - ein gewichtiger Beitrag zur Befähigung, Menschenrechte mit Respekt vor Diversität zu verwirklichen.

Zum anderen ist es Bildungspflicht der Schule, dass Kinder mit Behinderungen auch die Befähigung erwerben, an kinder- und menschenrechtlichen Projekten aktiv, kenntnisreich und verantwortlich mitzuwirken, die die Schule betreibt: keine Gewalt, keine Ausgrenzung, gegen Rassismus oder was immer das Thema sein mag. Die Zusicherung der Schule, das Recht auf Inklusion umzusetzen, verlöre an Glaubwürdigkeit, wenn die Schule nur den Kindern mit Behinderungen und nicht für kindermenschenrechtliche Probleme insgesamt offen wäre, denn die Konzentration auf nur eine Gruppe oder ein Problemfeld weckt den Verdacht, Wohlwollen nur selektiv aufzubringen und nicht die menschenrechtlichen Ansprüche insgesamt ernst zu nehmen. Immer ist die Aufgabenverteilung unter Erwachsenen und Kindern gut zu überlegen. Jedoch sollten Kinder mit Behinderungen auch Aufgaben übernehmen können, die ihre Fähigkeiten herausfordern und ihnen das „verantwortungsbewusste Leben“ in Gesellschaft und Staat ermöglichen.

\section{Inklusive Schulentwicklung: Die Schule als Menschenrechtsinstitution}

Inklusion ist nicht halb zu haben. Die Pflicht, Kindern mit Behinderungen inklusive Bildung zu bieten, ist nur zu erfüllen, wenn Schule ihren Auftrag nicht darauf reduziert, ihnen kognitive Lernprozesse zu erschließen, sondern sie als junge Menschen annimmt, die das unveräußerliche Recht besitzen, aktive Mitglieder ihrer sozialen Umwelt und der Gesellschaft zu sein.

Wer übergeht, dass die Staaten der Welt sich verpflichtet haben, in ihren Schulen diesen menschenrechtlichen Bildungsauftrag zu erfüllen, wird von den nun endlich für Aufnahme in Regelschulen fordernden Kindern mit Behinderungen daran erinnert, dass Kinder mehr brauchen als fachliche Kompetenz. Sie haben den verbürgten Anspruch, Mitbürger ${ }^{*}$ innen der schulischen Bildungsgemeinschaft 
von jungen und erwachsenen Menschen zu werden, weil die Probleme des Zusammenlernens und -lebens in der Schule bereits präsentieren, was sie ihr Leben lang herausfordern wird: gutes Leben ohne Exklusion und Ungerechtigkeit zu sichern. Verletzungen des menschenrechtlichen Status dieser Kinder wird auch ihren Bildungsprozessen schaden.

Diese Verpflichtung, Schule als Einrichtung zu begreifen, die Fähigkeiten vermittelt, um gemeinsames Leben auf der Grundlage der Menschenrechte zu gestalten, lässt sich nicht durch einige periphere Ergänzungen verwirklichen. Es geht um eine anders zentrierte Schule. Sie muss Vieles weiterführen, was unsere Bildungstradition mit guten Gründen der Schule zugewiesen hat. Sprachen, Literatur, Naturwissenschaften, Mathematik sind unverzichtbar, um an der Entwicklung des menschlichen Bewusstseins und der Suche nach Sinn teilzunehmen. Die Themen dieser Fächer müssen jedoch den Problemen näher sein, die uns heute bedrängen.

Entscheidend ist, dass zusätzliche Themen, Arbeitsweisen und Verfahren in die Schule, in Unterricht und Schulleben, aufgenommen werden, um Menschen zu befähigen, gemeinsam sachlich, kritisch-reflexiv und Rechte achtend gute Lebensformen zu entwickeln. Diese andere Schule unterrichtet nicht einige Stunden über Menschenrechte von Kindern und Erwachsenen, sondern ist eine Menschenrechtsinstitution. Kinder mit Behinderungen werden nicht inkludiert, sondern sie sind da.

Hilfskonzepte für definierte Gruppen erzeugen die Gefahr, eigene Entscheidungsräume dieser Menschen zu schmälern - gerade bei Menschen mit Behinderungen eine prekäre Situation. ${ }^{10}$ Erst die Schule, die sich der Umsetzung der Menschenrechte insgesamt verschreibt, bietet Kindern mit Behinderungen Mitwirkung, die sie nicht ausgrenzt. Als engagierte Mitwirkende an allen Themen können Kinder und Jugendliche wahrnehmen, dass nicht Hilfsmaßnahmen für einzelne Gruppen organisiert werden, sondern ihre Schule auf der menschenrechtlichen Verpflichtung aufbaut, die Verschiedenheit der Ausgangslagen, Sichtweisen und Interessen aller Schülerinnen als junge Menschenrechtssubjekte zu berücksichtigen.

Schulen können das leisten, wie zahlreiche Schulen und Schulnetzwerke beweisen, die auf Initiative von engagierten Schulleitungen oder Gruppen von Lehrpersonen, oft unterstützt von Stiftungen oder Bürgerorganisationen, sich auf diesen Weg begeben haben. Das verdient Anerkennung und Schulpreise, aber kann nicht die Pflicht der Staaten ersetzen, alle Bildungsstätten zu Menschenrechtsinstitutionen zu entwickeln, in denen jedes Kind seine Rechte genießt.

10 Die Menschenrechtskonventionen sprechen stets von Unterstützung oder Beistand (support, assistance), um durch die Wortwahl auszudrücken, dass nicht an Stelle einer Person gehandelt, sondern diese Person in ihrer Kompetenz gestärkt oder ihr Begleitung angeboten wird. 


\section{Literatur}

Ausschuss für die Rechte des Kindes (2006). Allgemeine Bemerkung Nr. 9: Die Rechte von Kindern mit Behinderungen. http://www.un.org/Depts/german/menschenrechte/crc-cgc9.pdf. Zugegriffen: 09.09.2016.

Bauer, J.-K., \& Feibig, M. (2013). Selbstwertstärkung durch Kinderrechtsbildung bei Kindern einer Förderschule. Eine exemplarische Fallstudie in Brandenburg. Master-These im Studiengang „Soziale Arbeit als Menschenrechtsprofession“ am Zentrum für postgraduale Studien Sozialer Arbeit Berlin.

Cremer, H. (2011). Menschenrechtsverträge als Quelle von individuellen Rechten. Innerstaatliche Geltung und Anwendbarkeit von Menschenrechtsverträgen am Beispiel der UN-Kinderrechtskonvention (KRK). Anwaltsblatt, 61, 159-165.

Krappmann, L., \& Petry, C. (Hrsg.). (2016). Worauf Kinder und Jugendliche ein Recht haben - Kinderrechte, Demokratie und Schule. Schwalbach i. T.: Debus Pädagogik.

Nussbaum, M. (2015). Fähigkeiten schaffen. Freiburg: Verlag Karl Alber.

Ständige Konferenz der Kultusminister der Länder (2000/1980). Empfehlung der Kultusministerkonferenz zur Förderung der Menschenrechtserziehung in der Schule. http://kmk. org/fileadmin/veroeffentlichungen_beschluesse/1980/1980_12_04_Menschenrechtserziehung.pdf. Zugegriffen: 09.09.2016. 